\title{
Metabolic and testicular effects of the long-term administration of different high-fat diets in adult rats
}

\author{
Pamella Campos-Silva ${ }^{1}$, Angelica Furriel ${ }^{1}$, Waldemar S. Costa ${ }^{1}$, Francisco J. B. Sampaio ${ }^{1}$, Bianca M. Gregório ${ }^{1}$ \\ ${ }^{1}$ Unidade de Pesquisa Urogenital, Centro Biomédico da Universidade Estadual do Rio de Janeiro, Brasil
}

\section{ABSTRACT}

Purpose: To evaluate the effects of different high-fat diets on body mass, carbohydrate metabolism and testicular morphology in rats seven months old.

Materials and Methods: Male Wistar rats were divided into four groups: SC (standard chow), HF-S (high fat diet rich in saturated fatty acids), HF-P (high fat diet rich in polyunsaturated fatty acids), HF-SP (high fat diet rich in saturated and polyunsaturated fatty acids). The rats were fed for 16 weeks. Blood samples, testes and genital fat deposits were collected for analysis. Data were analyzed by one-way ANOVA and Bonferroni post hoc test, considering $\mathrm{p}<0.05$ as statistically significant.

Results: Different high-fat diets promoted an increase in the body mass $(\mathrm{p}<0.0001)$. The genital fat deposits were higher in the high-fat groups (HF-S, HF-P, HF-SP) ( $p=0.0004)$. Regarding serum parameters, the animals in the HF-S and HF-SP groups presented hyperglycemia $(p=0.0060)$, hyperinsulinemia $(p=0.0030)$ and hypercholesterolemia $(p=0.0021)$. All of the hyperlipidemic groups showed hyperleptinemia ( $p=0.0019)$. Concerning the testis, the HF-S group showed a reduction on the seminiferous epithelium height $(p=0.0003)$ and cell proliferation $(p=0.0450)$. Seminiferous tubule diameter was lower in the HF-SP than in the SC group ( $p=0.0010)$.

Conclusions: The high fat diet administration, independent of the lipid quality, promotes overweight. Diet rich in saturated fatty acids (lard) alters the carbohydrate metabolism and the testicular morphology with reductions of seminiferous epithelium height, seminiferous tubule diameter and cell proliferation which could be related to a disturbance of spermatogenesis.

\section{ARTICLE INFO}

Key words:

Obesity; Fatty Acids, Unsaturated;

Fatty Acids; Testis; Rats, Wistar

Int Braz J Urol. 2015; 41: 569-75

Submitted for publication:

May 15, 2014

Accepted after revision:

October 19, 2014

\section{INTRODUCTION}

The World Health Organization (WHO) estimates that more than half a billion adults are obese and approximately three million people die as a result of obesity per year (1). It is know that an excess of lipid intake, regardless of the type of fat, causes obesity. Obesity is normally associated with a higher incidence of insulin resistance, type 2 diabetes mellitus, hypertension, dyslipidemia, some types of cancer and certain metabolic and reproductive disorders (2).

In recent years, several studies have shown an inverse relationship between the body mass index (BMI) and hyperleptinemia in male reproductive parameters. In obese men, leptin can also act to lower the testosterone level, implying in hypogonadism (3). The fat rich-diets affect the organization of the plasma membrane. One of the consequences in the testes is the altered availabili- 
ty of gonadotropin receptors, such as luteinizing hormone (LH) and follicle stimulating hormone (FSH), comprising testosterone production by the Leydig cells (interstitial) and spermatogenesis by the cells of the seminiferous tubules, respectively (4).

There are few data relating the effects of saturated and polyunsaturated fatty acids upon the testicular components in rats. The aim of the present study was to evaluate the body mass, carbohydrate metabolism and testicular morphology in Wistar rats at seven months old that were fed with different high-fat diets (rich in saturated and/or polyunsaturated fatty acids).

\section{MATERIALS AND METHODS}

\section{Experimental protocol}

This study was approved by the Ethics Committee for the Care and Use of Experimental Animals of the Institute of Biology, CEUA0272012) and followed the guidelines suggested by the Brazilian College of Animal Experimentation (COBEA).

Male Wistar rats aged three months were divided into four experimental groups according to the lipid content of their diet: SC (standard chow, $n=9$ ), HF-S (high fat diet rich in saturated fatty acids, based on lard, $n=10$ ), HF-P (high fat diet rich in polyunsaturated fatty acids, based on canola oil, $\mathrm{n}=10$ ) and HF-SP (high fat diet rich in saturated and polyunsaturated fatty acids, $n=10$ ). The SC diet (14\% protein, 76\% carbohydrates and 10\% lipids with a total energy of $15.9 \mathrm{~kJ} / \mathrm{g}$ ) and the different high-fat diets (14\% protein, 36\% carbohydrates and 50\% fat with a total energy of $20.9 \mathrm{~kJ} / \mathrm{g}$ ) were prepared following the recommendations of the AIN-93M (5). The diets were made by Pragsolutions ${ }^{\circledR}$ (www. pragsolucoes.com.br) and the high fat diets included added lard (saturated fatty acid) and/or canola oil (polyunsaturated fatty acid).

The control and HF groups received the diets SC, HF-S, HF-P and HF-SP over 16 weeks, from three months until seven months of age. The food intake was recorded daily. Body mass (BM) was monitored weekly until the end of the experiment. The animals were placed in an appropriate environment with a temperature of $21 \pm 2^{\circ} \mathrm{C}$ and controlled light cycle (12-12 h light/dark), with free access to water and food.

\section{Euthanasia of animals}

After 12 hours of fasting, the animals were anesthetized intraperitoneally with sodium pentobarbital and killed at seven months of age. Blood samples were collected from the heart (right atrium) by cardiac puncture, for the serum analysis. The testes and genital fat pad were dissected, weighed and fixed.

Serum biochemistry and hormone levels

Serum was separated by centrifugation (3000 rpm, for $8 \mathrm{~min}$ ) at room temperature and stored at $-20^{\circ} \mathrm{C}$ for the serum analysis. The glucose (monoreagent-K082), triglyceride (monoreagent-K117) and total cholesterol (monoreagent-K083) concentrations were measured by a colorimetric assay (Bioclin Systems II ${ }^{\circledR}$, Quisaba, Bioclin, Belo Horizonte, MG, Brazil). The serum analyses for insulin, leptin and testosterone were performed using the following commercially available enzyme-linked immunosorbent assay (ELISA) kits: rat/mouse insulin kit (Millipore-Cat. EZRMI-13 k - St Charles, MO, USA), rat leptin kit (Millipore-Cat. EZRL-83K, St Charles, MO, USA) and general testosterone kit (Uscn-Cat. E90458Ge - Wuhan, China). All samples were analyzed in duplicate with an intra-assay coefficient of variation of 1.4\%.

\section{Immunohistochemistry}

Sections of the testes at a thickness of $5 \mu \mathrm{m}$ were deparaffinized, and antigen retrieval was performed with TRIS-EDTA ( $\mathrm{pH}$ 9.0) for 12 hours (overnight) at $60^{\circ} \mathrm{C}$. Then, an endogenous peroxidase blockade was made with hydrogen peroxidase 3\%, and nonspecific binding was inhibited. The sections were incubated with anti-PCNA antibody (PC10, Ref: 180110, Invitrogen, Camarillo, CA, USA), and the immunoreaction was amplified with the biotin-streptavidin system kit (Ref: 859643, Invitrogen, Frederick, MD, USA). Immunostaining was visualized after incubation of the sections with 3 , 3-diaminobenzidine tetrachloride (Ref: 859643, In- 
vitrogen, Frederick, MD, USA) and counterstaining with Mayer's hematoxylin.

\section{Testis quantitative study}

The testes were fixed in Bouin's solution for 24 hours and $1.27 \mathrm{M}$ formaldehyde in $0.1 \mathrm{M}$ phosphate buffer, $\mathrm{pH} 7.2$ for $48 \mathrm{~h}$ at room temperature, after was embedded in Paraplast plus ${ }^{\circledR}$ (Sigma-Aldrich C., St. Louis, MO, USA). Subsequently, the material was sectioned at a thickness of $5 \mu \mathrm{m}$ and stained with hematoxylin and eosin. Digital images from the testes were obtained by an Olympus BX51 light microscope (Tokyo, Japan) coupled to a digital camera (Olympus DP70-Tokyo, Japan). The quantitative evaluation was performed using the software Image ${ }^{\circledR}$ (Image Processing and Analysis in Java). After calibration, the seminiferous tubule diameter and seminiferous epithelium height were determined with the "straight line selections" tool. These morphometric analyses were performed with a $10 \mathrm{X}$ objective for the seminiferous tubule diameter and a 20X objective for the seminiferous epithelium height. The rate of cell proliferation was measured with the "cell counter" tool, and then the "free hand selections" tool was used to delimit the area quantified. Photomicrographs were obtained using an objective of 40X. For these analyses, 25 fields/animal were also assessed.

\section{Biochemical Analysis}

The samples were fixed in cold acetone for 24 hours at $4{ }^{\circ} \mathrm{C}$. Then, the material was cleaved ( $2 \mathrm{~mm} \times 2 \mathrm{~mm}$ ) and submitted to two soaks of 24 hours each in $40 \mathrm{~mL}$ of chloroform/methanol (2:1, $\mathrm{v} / \mathrm{v})$ at room temperature. The material was incubated at $60^{\circ} \mathrm{C}$ for 30 minutes. Thus, 5 to $15 \mathrm{mg}$ of testicular dry tissue was defatted and hydrolyzed in $6 \mathrm{~N} \mathrm{HCl}$ for 18 hours at $118^{\circ} \mathrm{C}$. The dosage was determined using neutralized hydrolyzates by the chloramin $\mathrm{T}$ method (6).

\section{Data analysis}

The data were reported as the mean \pm standard deviation (SD). Differences between groups were calculated by the analysis of variance (one-way ANOVA) and Bonferroni post hoc test. In all cases, a $p$-value $\leq 0.05$ was considered statistically significant (GraphPad Prism version 5.03 for Windows-GraphPad Software, San Diego, CA, USA).

\section{RESULTS}

Food intake and body mass

Throughout the experiment, the HF-S, HF-P and HF -SP groups showed an increase of food intake. This pattern was accompanied by a significant increase in the body mass of the HF-S (529.30 $\pm 57.39 \mathrm{~g}), \mathrm{HF}-\mathrm{P}(546.40 \pm 40.13 \mathrm{~g})$ and HF-SP $(532.90 \pm 48.27 \mathrm{~g})$ groups compared with the SC group (424.22 $\pm 40.29 \mathrm{~g}, \mathrm{p}<0.0001)$.

\section{Genital fat pad}

Animals fed the HF diet had a greater epididymal fat mass (HF-S: $12.79 \pm 4.05 \mathrm{~g}$, HF-P: $14.64 \pm 4.75 \mathrm{~g}, \mathrm{HF}-\mathrm{SP}: 14.54 \pm 2.12 \mathrm{~g})$ than those in the SC group $(7.65 \pm 2.70 \mathrm{~g}, \mathrm{p}=0.0004)$, corresponding to increases of $67 \%, 91 \%$ and $90 \%$ of the compartment, respectively.

\section{insulin values)}

Carbohydrate metabolism (glucose and

The animals in the HF-S and HF-SP groups showed hyperglycemia $(\mathrm{p}=0.0060)$ and hyperinsulinemia $(\mathrm{p}=0.0030)$ (Table-1).

Lipid profile, leptin and testosterone levels The values of total cholesterol were higher in the HF-S and HF-SP groups than in the $S C$ group $(p=0.0021)$. All animals that received the HF diets showed hyperleptinemia $(p=0.0019)$. The triglyceride and testosterone levels did not differ among the groups (Table-1).

\section{Testes parameters}

The testicular mass did not differ among the groups. Figure-1 shows the seminiferous epithelium height and seminiferous tubule diameter, and Figure- 2 shows the proliferation of a spermatogenic cell line in the testes of the different groups. The SC, HF-P and HF-SP groups showed the highest seminiferous epithelium compared with the HF-S group ( $p=0.0003)$. Regarding the seminiferous tubule diameter, there was a decre- 
Table 1 - Carbohydrate metabolism (glucose and insulin values), lipid profile (triglycerides and total cholesterol values), leptin levels, testosterone levels and testes parameters in rats fed for 16 weeks.

\begin{tabular}{lcccc}
\hline Serum Analyses & SC $(\mathrm{n}=9)$ & HF-S $(\mathrm{n}=10)$ & $H F-P(\mathrm{n}=10)$ & HF-SP $(\mathrm{n}=10)$ \\
\hline Glucose $(\mathrm{mmol} / \mathrm{L})$ & $7.87 \pm 1.62$ & $10.62 \pm 2.36^{\mathrm{a}}$ & $9.85 \pm 1.61$ & $11.09 \pm 1.65^{\mathrm{a}}$ \\
Insulin $(\mu \mathrm{LU} / \mathrm{ml})$ & $1.49 \pm 0.41$ & $2.75 \pm 0.45^{\mathrm{a}}$ & $2.15 \pm 0.83$ & $2.85 \pm 0.92^{\mathrm{a}}$ \\
Triglycerides $(\mathrm{mg} / \mathrm{dL})$ & $86.29 \pm 23.68$ & $95.63 \pm 8.60$ & $83.63 \pm 17.52$ & $84.25 \pm 5.04$ \\
Total Cholesterol $(\mathrm{mg} / \mathrm{dL})$ & $80.56 \pm 11.75$ & $104.80 \pm 12.95^{\mathrm{a}}$ & $100.10 \pm 10.38$ & $105.20 \pm 19.65^{\mathrm{a}}$ \\
Leptin $(\mathrm{ng} / \mathrm{mL})$ & $6.79 \pm 3.73$ & $12.04 \pm 1.02^{\mathrm{a}}$ & $11.49 \pm 1.73^{\mathrm{a}}$ & $11.63 \pm 1.61^{\mathrm{a}}$ \\
Testosterone $(\mathrm{ng} / \mathrm{mL})$ & $5.48 \pm 0.83$ & $4.73 \pm 1.14$ & $4.28 \pm 1.25$ & $5.11 \pm 0.89$ \\
Testes Parameters & $\mathrm{SC}(\mathrm{n}=9)$ & $\mathrm{HF}-\mathrm{S}(\mathrm{n}=10)$ & $\mathrm{HF}-\mathrm{P}(\mathrm{n}=10)$ & $\mathrm{HF}-\mathrm{SP}(\mathrm{n}=10)$ \\
Testicular mass $(\mathrm{g})$ & $1.46 \pm 0.09$ & $1.51 \pm 0.07$ & $1.52 \pm 0.07$ & $1.53 \pm 0.08$ \\
Seminiferous epithelium & $44.84 \pm 1.46$ & $41.66 \pm 2.60^{\mathrm{a}}$ & $46.12 \pm 2.24 \mathrm{~b}$ & $44.92 \pm 1.92^{\mathrm{b}}$ \\
height $(\mu \mathrm{m})$ & & & & \\
Seminiferous tubule & $310.60 \pm 6.99$ & $303.30 \pm 8.26$ & $297.90 \pm 10.15$ & $291.40 \pm 11.46 \mathrm{a}$ \\
diameter $(\mu \mathrm{m})$ & & & & \\
Cell proliferation $\left(\mu \mathrm{m} / \mathrm{mm}^{2}\right)$ & $3.24 \times 10^{-3} \pm 0.83 \times 10^{-3}$ & $2.25 \times 10^{-} 3 \pm 0.57 \times 10^{-3} \mathrm{a}$ & $2.89 \times 10^{-3} \pm 0.23 \times 10^{-3}$ & $2.75 \times 10^{-3} \pm 0.36 \times 10^{-3}$ \\
Collagen $(\mu \mathrm{g} / \mathrm{mg})$ & $2.09 \pm 0.23$ & $2.08 \pm 0.21$ & $1.94 \pm 0.68$ & $2.07 \pm 0.37$ \\
\hline
\end{tabular}

ase in the HF-SP group compared with the SC group $(\mathrm{p}=0.0010)$. Cell proliferation was reduced in HF-S group compared with the SC group $(\mathrm{p}=0.0450)$. The collagen level did not differ among the groups (Table-1).

\section{DISCUSSION}

Comparing the metabolic effects triggered by different high fat diets, diets based on saturated fatty acids and cholesterol are believed to induce deleterious effects on the body mass and metabolism and to predispose to the development of insulin resistance, whereas diets based on polyunsaturated fatty acids are believed to exert opposite effects (7). In the present study, both diets were able to promote an increase in body mass. Hariri, Gougeon et al. (2010) also observed overweight in animals fed with a high fat diet, regardless of the lipid quality (8).

In addition to the increase in body mass, all HF groups presented an increase in genital fat deposits, confirming the overweight. The HF-S and HF-SP groups showed increased serum levels of glucose, insulin and total cholesterol. Thus, the consumption of saturated fat at different concentrations (50\% and 25\% of the total energy in the diet) was capable of causing metabolic changes. In humans, recent evidence has correlated metabolic syndrome with hypogonadism (9). Nascimento, Barbosa-da-Silva et al. (2010) also reported that C57BL/6 mice developed obesity, insulin resistance and lipid profile alterations when fed a diet rich in saturated fatty acids (10). Obesity and metabolic syndrome or one of its components (hyperlipidemia) can cause oxidative stress (11). The testicular tissues and spermatozoa are very sensitive to reactive oxygen species attack and lipid peroxidation (12). Some studies have revealed that oxidative stress resulted in sperm membrane lipid peroxidation with impairment in sperm motility and sperm-oocyte interaction (13).

In our study, simultaneous with the increase of white adipose tissue, the HF diets raise the 
Figure-1 - Photomicrographs of the seminiferous tubule diameter and seminiferous epithelium height.

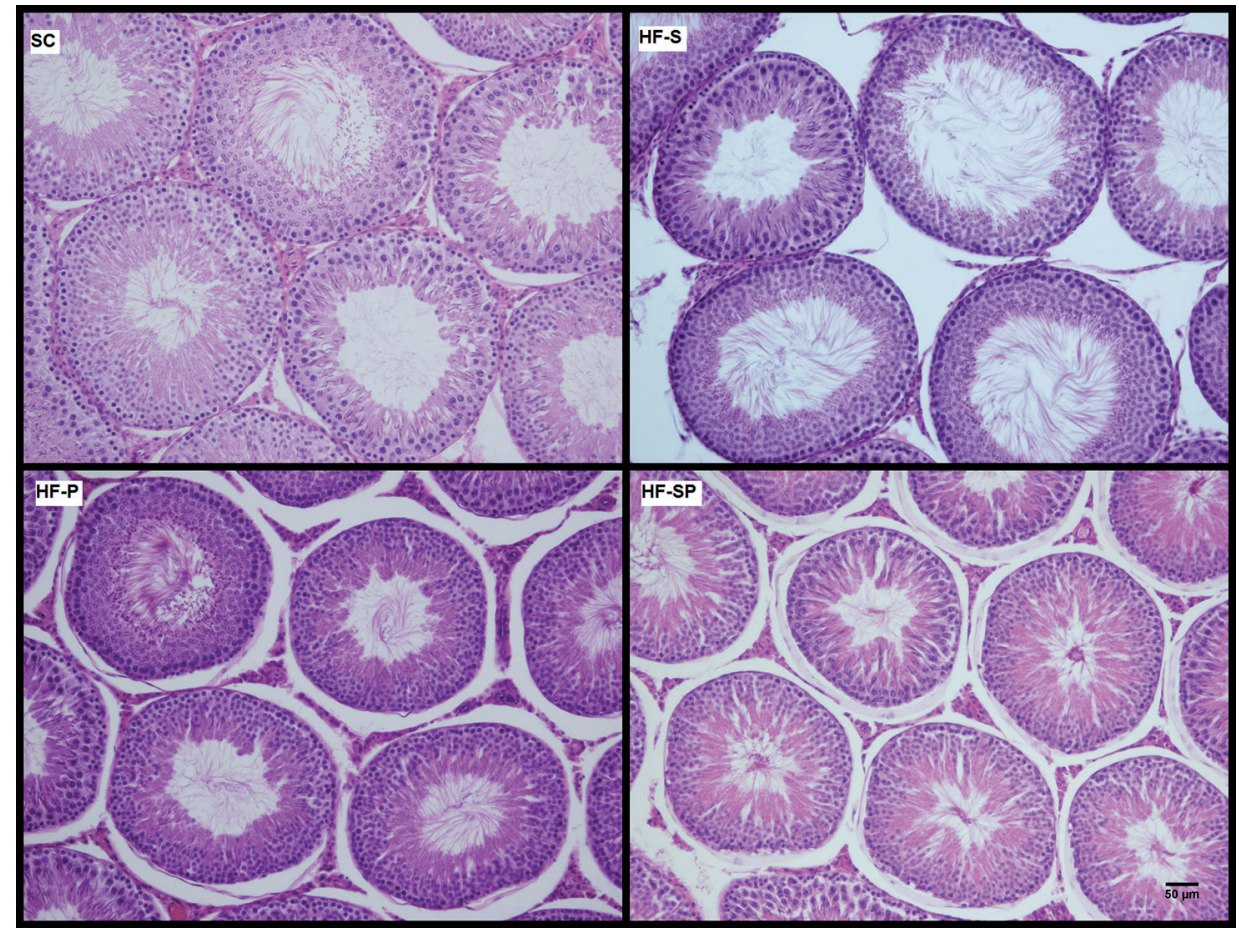

SC (standard chow); HF-S (high-fat diet rich in saturated fatty acids); HF-P (high-fat diet rich in polyunsaturated fatty acids) and HF-SP (high-fat diet rich in saturated and polyunsaturated fatty acids). The data are reported as mean $\pm S D$. The differences were tested by the one-way analysis of variance (ANOVA) and the Bonferroni post hoc test, $p<0.05$. The HF-S and HF-SP groups showed a reduction in the seminiferous epithelium height and seminiferous tubule diameter, respectively, which may affect sperm production. H-E staining 200x.

serum levels of leptin, consistent with the observation that white adipose tissue is the main source of leptin in adult animals (14). Hyperleptinemia appears to be involved in the reduction of serum testosterone levels. Vigueras-Villasenor, Rojas-Castaneda et al. (2011) observed a reduction in testosterone levels in Sprague-Dawley rats fed a HF diet from weaning at 90 days (15). This work did not show a decrease in the testosterone levels, although there were increased levels of leptin. The animals' body mass may not have been sufficient to produce a significant decrease in the serum testosterone levels.

One of the parameters used for the evaluation of spermatogenesis is the seminiferous tubule diameter (16). In our work, the seminiferous tubule diameter was reduced in the HF-SP group, indicating a decrease in spermatozoa production. Lard administration in the diet of the HF-SP group may have been the cause of this reduction. With respect to the seminiferous epithelium height, the HF-S group showed a significant decrease compared with the control group. However, the HF-P and HF -SP groups showed results similar to those of the SC group. Cholesterol is known to be part of the composition of animal cell membranes, promoting the rigidity of the plasma membrane when in excess (17). The expected result, therefore, is that the increased intake of polyunsaturated fatty acids may improve the physico-chemical properties of the plasma membranes and thus promote spermatogenesis. Sebokova, Garg et al. (1990) observed the accumulation of cholesterol in the plasma membrane of germ cells after the administration of hypercholesterolemic diets (18).

Cell proliferation was significantly reduced in the HF-S group, showing that some stage of spermatogenesis was affected by cholesterol. 
Figure-2 - Photomicrographs of the proliferation of a spermatogenic cell line in animals.

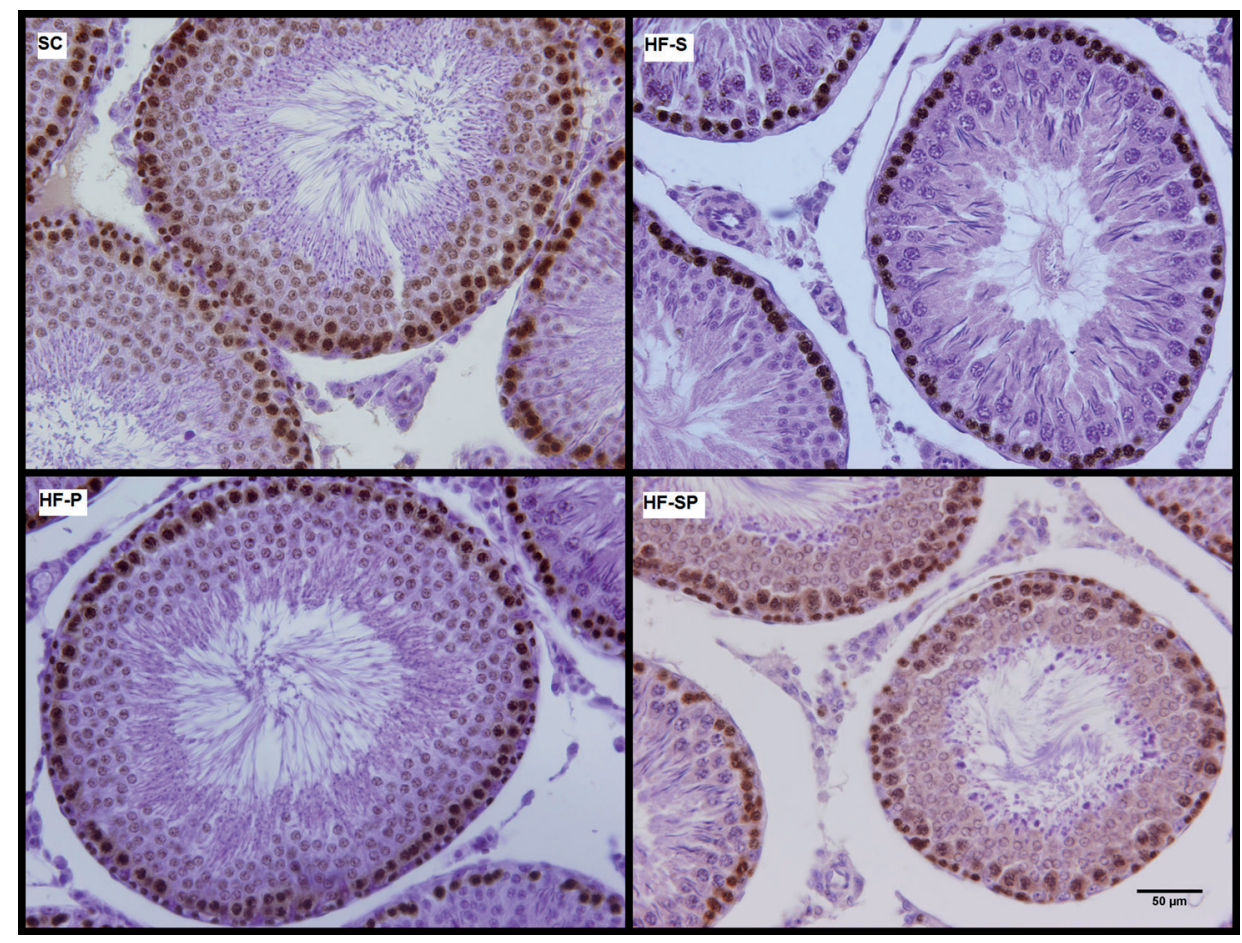

SC (standard chow); HF-S (high-fat diet rich in saturated fatty acids), HF-P (high-fat diet rich in polyunsaturated fatty acids) and HF-SP (high-fat diet rich in saturated and polyunsaturated fatty acids). The data are reported as the mean $\pm S D$. The differences were tested by the one-way analysis of variance (ANOVA) and the Bonferroni post hoc test, $p<0.05$. The HF-S group showed a reduction in the proliferation of the spermatogenic cell line, which can affect sperm production. Immunohistochemical staining for PCNA 400x.

Purohit and Daradka (1999) obtained the same result in hyperlipidemic rats (19). These authors noted that spermatogenesis was arrested at primary spermatocyte stage because few secondary spermatocytes were observed. These observations are consistent with our results.

Biancalana et al. (2010) observed significant changes in the hydroxyproline content in the tendons of obese mice (20). Collagen changes in the urogenital system of obese mouse have not been described. In the present study, the amount of hydroxyproline in the rats' testes was similar in all groups, showing that the different fat diets did not affect the amount of collagen in the obese rat testes.

\section{CONCLUSIONS}

The high fat diet administration, independent of the lipid quality, promotes overweight. However, a diet rich in saturated fatty acids (lard) alters the carbohydrate metabolism and testicular morphology, with reductions of the seminiferous epithelium height, seminiferous tubule diameter and proliferation of cells of a spermatogenic lineage, which could be related to a disturbance of spermatogenesis.

\section{ABBREVIATIONS}

LH = luteinizing hormone

$\mathrm{FSH}=$ follicle stimulating hormone

CEUA = Ethics Committee for the Care and Use of Experimental Animals

IBRAG = Biology Institute of Roberto Alcantara Gomes

COBEA = Brazilian College of Animal Experimentation

AIN-93 = American Institute of Nutrition

\section{CONFLICT OF INTEREST}

None declared. 


\section{REFERENCES}

1. World Health Organization. Noncommunicable diseases country profiles. Available at: http://www.who.int/nmh/ publications/ncd_profiles2011. Accessed march 06, 2013.

2. Fernandez CD, Bellentani FF, Fernandes GS, Perobelli JE, Favareto AP, Nascimento AF, et al. Diet-induced obesity in rats leads to a decrease in sperm motility. Reprod Biol Endocrinol. 2011;9:32.

3. Michalakis K, Mintziori G, Kaprara A, Tarlatzis BC, Goulis $D G$. The complex interaction between obesity, metabolic syndrome and reproductive axis: a narrative review. Metabolism. 2013;62:457-78.

4. Mah PM, Wittert GA. Obesity and testicular function. Mol Cell Endocrinol. 2010;316:180-6.

5. Reeves PG, Nielsen FH, Fahey GC Jr. AIN-93 purified diets for laboratory rodents: final report of the American Institute of Nutrition ad hoc writing committee on the reformulation of the AIN-76A rodent diet. J Nutr. 1993;123:1939-51.

6. Bergman I, Loxley R: Two improved and simplified methods for the spectrophotometric determination of hydroxyproline. Anal Biochem. 1963; 35: 1961-5.

7. Cascio G, Schiera G, Di Liegro I. Dietary fatty acids in metabolic syndrome, diabetes and cardiovascular diseases. Curr Diabetes Rev. 2012;8:2-17.

8. Hariri N, Gougeon R, Thibault L. A highly saturated fat-rich diet is more obesogenic than diets with lower saturated fat content. Nutr Res. 2010;30:632-43.

9. Stanworth RD, Jones TH. Testosterone in obesity, metabolic syndrome and type 2 diabetes. Front Horm Res. 2009;37:7490.

10. Nascimento FA, Barbosa-da-Silva S, Fernandes-Santos C, Mandarim-de-Lacerda CA, Aguila MB. Adipose tissue, liver and pancreas structural alterations in C57BL/6 mice fed high-fat-high-sucrose diet supplemented with fish oil (n-3 fatty acid rich oil). Exp Toxicol Pathol. 2010;62:17-25.

11. Hansel B, Giral P, Nobecourt E, Chantepie S, Bruckert E, Chapman MJ, et al. Metabolic syndrome is associated with elevated oxidative stress and dysfunctional dense highdensity lipoprotein particles displaying impaired antioxidative activity. J Clin Endocrinol Metab. 2004;89:4963-71.
12. Farias JG, Puebla M, Acevedo A, Tapia PJ, Gutierrez E, Zepeda $A$, et al. Oxidative stress in rat testis and epididymis under intermittent hypobaric hypoxia: protective role of ascorbate supplementation. J Androl. 2010;31:314-21.

13. Codoñer-Franch P, Tavárez-Alonso S, Murria-Estal R, MegíasVericat J, Tortajada-Girbés M, Alonso-Iglesias E. Nitric oxide production is increased in severely obese children and related to markers of oxidative stress and inflammation. Atherosclerosis. 2011;215:475-80.

14. Sakurai T, Ogasawara J, Kizaki T, Sato S, Ishibashi $Y$, Takahashi $M$, et al. The effects of exercise training on obesity-induced dysregulated expression of adipokines in white adipose tissue. Int J Endocrinol. 2013;2013:801743.

15. Vigueras-Villaseñor RM, Rojas-Castañeda JC, ChávezSaldaña M, Gutiérrez-Pérez 0, García-Cruz ME, CuevasAlpuche 0 , et al. Alterations in the spermatic function generated by obesity in rats. Acta Histochem. 2011;113:21420.

16. Attal J, Courot M. Développement testiculaire et établissement de la spermatogénèse chez le taureau. Ann. Biol. Anim. Bioch. Biophys., 1963;3:219-41.

17. Demel RA, De Kruyff $B$. The function of sterols in membranes. Biochim Biophys Acta. 1976;457:109-32.

18. Sebokova E, Garg ML, Wierzbicki A, Thomson AB, Clandinin MT. Alteration of the lipid composition of rat testicular plasma membranes by dietary $(n-3)$ fatty acids changes the responsiveness of Leydig cells and testosterone synthesis. J Nutr. 1990;120:610-8.

19. Purohit A, Daradka HM. Effect of mild hyperlipidaemia on testicular cell population dynamics in albino rats. Indian $\mathrm{J}$ Exp Biol. 1999;37:396-8.

20. Biancalana A, Veloso LA, Gomes L. Obesity affects collagen fibril diameter and mechanical properties of tendons in Zucker rats. Connect Tissue Res. 2010;51:171-8.

Correspondence address:

Bianca Martins Gregório, MD

Unidade de Pesquisa Urogenital

Centro Biomédico, Universidade do Estado do Rio de Janeiro Av. 28 de setembro 87 Rio de Janeiro, RJ, 20551-030, Brazil Telephone: + 5521 2868-8021 E-mail: biancamgregorio.uerj@gmail.com 